\title{
Defined Activity Repeat Duration
}

National Cancer Institute

\section{Source}

National Cancer Institute. Defined Activity Repeat Duration. NCI Thesaurus. Code C93754.

The period of time over which the activity is repeated. 